\title{
Non-Hamiltonian generalizations of the dispersionless 2DTL hierarchy
}

\author{
L.V. Bogdanov*
}

March 9, 2022

\begin{abstract}
We consider two-component integrable generalizations of the dispersionless 2DTL hierarchy connected with non-Hamiltonian vector fields, similar to the Manakov-Santini hierarchy generalizing the dKP hierarchy. They form a one-parametric family connected by hodograph type transformations. Generating equations and Lax-Sato equations are introduced, a dressing scheme based on the vector nonlinear Riemann problem is formulated. The simplest two-component generalization of the dispersionless 2DTL equation is derived, its differential reduction analogous to the Dunajski interpolating system is presented. A symmetric two-component generalization of the dispersionless elliptic 2DTL equation is also constructed.
\end{abstract}

\section{Introduction}

Recently S.V. Manakov and P.M. Santini introduced a two-component system generalizing the dispersionless KP equation to the case of non-Hamiltonian vector fields in the Lax pair [1,2],

$$
\begin{aligned}
& u_{x t}=u_{y y}+\left(u u_{x}\right)_{x}+v_{x} u_{x y}-u_{x x} v_{y} \\
& v_{x t}=v_{y y}+u v_{x x}+v_{x} v_{x y}-v_{x x} v_{y}
\end{aligned}
$$

and the Lax pair is

$$
\begin{aligned}
& \partial_{y} \boldsymbol{\Psi}=\left(\left(\lambda-v_{x}\right) \partial_{x}-u_{x} \partial_{\lambda}\right) \boldsymbol{\Psi} \\
& \partial_{t} \boldsymbol{\Psi}=\left(\left(\lambda^{2}-v_{x} \lambda+u-v_{y}\right) \partial_{x}-\left(u_{x} \lambda+u_{y}\right) \partial_{\lambda}\right) \boldsymbol{\Psi}
\end{aligned}
$$

${ }^{*}$ L.D. Landau ITP RAS, Moscow, Russia, e-mail leonid@landau.ac.ru 
where $u, v$ are functions of $x, y, t$, and $\lambda$ plays a role of a spectral variable. For $v=0$ the system (1) reduces to the dKP (Khohlov-Zabolotskaya) equation

$$
u_{x t}=u_{y y}+\left(u u_{x}\right)_{x} .
$$

Respectively, the reduction $u=0$ gives an equation [3]

$$
v_{x t}=v_{y y}+v_{x} v_{x y}-v_{x x} v_{y} .
$$

The hierarchy related to this system was studied in $[4,5]$. It was demonstrated that the Manakov-Santini hierarchy represents a case $\mathrm{N}=1$ of a general $(\mathrm{N}+1)$-component hierarchy. This general hierarchy is connected with commutativity of $(\mathrm{N}+1)$-dimensional vector vields containing a derivative with respect to the spectral variable, with the coefficients of vector fields meromorphic in the complex plane of the spectral variable and having a pole only at one point (e.g., infinity, compare the Lax pair (2)). In this sense the Manakov-Santini hierarchy is a two-component one-point hierarchy, and generalizations of the dispersionless 2DTL hierarchy we are going to consider in this paper represent a two-component two-point case, when vector fields have poles at two points (say, zero and infinity).

Our starting point is the formalism of the works $[6,4,5]$, which we transfer to the two-point case having in mind the representation of the dispersionless 2DTL hierarchy given in $[7,8]$ and the results of the recent work [9], in which the dressing, the Cauchy problem and the behavior of solutions of the dispersionless 2D Toda equation were studied. We introduce generating equations and Lax-Sato equations and develop a dressing scheme based on vector nonlinear Riemann problem. We discover one-parametric freedom in generalizing the dispersionless 2DTL hierarchy, and describe hodograph type transformations connecting different generalizations.

The simplest two-component generalization of the dispersionless 2DTL equation reads

$$
\begin{aligned}
\left(\mathrm{e}^{-\phi}\right)_{t t} & =m_{t} \phi_{x y}-m_{x} \phi_{t y}, \\
m_{t t} \mathrm{e}^{-\phi} & =m_{t y} m_{x}-m_{x y} m_{t},
\end{aligned}
$$

and the Lax pair is

$$
\begin{aligned}
\partial_{x} \boldsymbol{\Psi} & =\left(\left(\lambda+\frac{m_{x}}{m_{t}}\right) \partial_{t}-\lambda\left(\phi_{t} \frac{m_{x}}{m_{t}}-\phi_{x}\right) \partial_{\lambda}\right) \Psi \\
\partial_{y} \boldsymbol{\Psi} & =\left(\frac{1}{\lambda} \frac{\mathrm{e}^{-\phi}}{m_{t}} \partial_{t}+\frac{\left(\mathrm{e}^{-\phi}\right)_{t}}{m_{t}} \partial_{\lambda}\right) \boldsymbol{\Psi}
\end{aligned}
$$


(the derivation is given below). For $m=t$ the system (5) reduces to the dispersionless 2DTL equation

$$
\left(\mathrm{e}^{-\phi}\right)_{t t}=\phi_{x y}
$$

Respectively, the reduction $\phi=0$ gives an equation [3]

$$
m_{t t}=m_{t y} m_{x}-m_{x y} m_{t} .
$$

System (5) doesn't preserve the symmetry of the dispersionless 2DTL equation with respect to $x, y$ variables, however, we also introduce a symmetric generalization of the d2DTL equation.

\section{Generalized dispersionless 2DTL hierarchy}

We generalize a picture of the dispersionless 2DTL hierarchy given by Takasaki and Takebe [7, 8], taking into account the results of the recent work [9], to the case of non-Hamiltonian vector fields, similar to the Manakov-Santini hierarchy, which generalizes the dispersionless KP hierarchy $[1,2,4,5]$. We consider formal series

$$
\begin{aligned}
& \Lambda^{\text {out }}=\ln \lambda+\sum_{k=1}^{\infty} l_{k}^{+} \lambda^{-k}, \quad \Lambda^{\text {in }}=\ln \lambda+\phi+\sum_{k=1}^{\infty} l_{k}^{-} \lambda^{k}, \\
& M^{\text {out }}=M_{0}^{\text {out }}+\sum_{k=1}^{\infty} m_{k}^{+} \mathrm{e}^{-k \Lambda^{+}}, \quad M^{\text {in }}=M_{0}^{\text {in }}+m_{0}+\sum_{k=1}^{\infty} m_{k}^{-} \mathrm{e}^{k \Lambda^{-}}, \\
& M_{0}=t+x \mathrm{e}^{\Lambda}+y \mathrm{e}^{-\Lambda}+\sum_{k=1}^{\infty} x_{k} \mathrm{e}^{(k+1) \Lambda}+\sum_{k=1}^{\infty} y_{k} \mathrm{e}^{-(k+1) \Lambda},
\end{aligned}
$$

where $\lambda$ is a spectral variable. Usually we suggest that 'out' and 'in' components of the series define the functions outside and inside the unit circle in the complex plane of the variable $\lambda$ respectively, with $\Lambda^{\text {in }}-\ln \lambda, M^{\text {in }}-M_{0}^{\text {in }}$ analytic in the unit disc, and $\Lambda^{\text {out }}-\ln \lambda, M^{\text {out }}-M_{0}^{\text {out }}$ analytic outside the unit disc and decreasing at infinity. For a function on the complex plane, having a discontinuity on the unit circle, by 'in' and 'out' components we mean the function inside and outside the unit disc. For two-component series we observe a natural convention $(A B)^{\text {in }}=A^{\text {in }} B^{\text {in }},(A B)^{\text {out }}=A^{\text {out }} B^{\text {out }}$, which corresponds to multiplication of respective functions on the complex plane. The coefficients of the series $\phi, m_{0}, l_{k}^{ \pm}, m_{k}^{ \pm}$are functions of times $t$, $x_{n}, y_{n}$. Usually for simplicity we suggest that only finite number of $x_{k}, y_{k}$ are not equal to zero. 
Generalized dispersionless 2DTL hierarchy is defined by the generating relation

$$
\left(\left(J_{0}\right)^{-1} \mathrm{~d} \Lambda \wedge \mathrm{d} M\right)^{\text {out }}=\left(\left(J_{0}\right)^{-1} \mathrm{~d} \Lambda \wedge \mathrm{d} M\right)^{\mathrm{in}},
$$

which may be considered as a continuity condition on the unit circle for the differential two-form (or just in terms of formal series), where $J_{0}$ is a determinant of Jacobi type matrix $J$,

$$
J=\left(\begin{array}{cc}
\lambda \partial_{\lambda} \Lambda & \partial_{t} \Lambda \\
\lambda \partial_{\lambda} M & \partial_{t} M
\end{array}\right)
$$

$J_{0}^{\text {out }}=1+O\left(\lambda^{-1}\right), J_{0}^{\text {in }}=1+\partial_{t} m_{0}+O(\lambda)$, and we suggest that $J_{0} \neq 0$; the differential $\mathrm{d}$ is given by

$$
\mathrm{d} f=\partial_{\lambda} f \mathrm{~d} \lambda+\partial_{t} f \mathrm{~d} t+\sum_{k=1}^{\infty} \frac{\partial f}{\partial x_{k}} \mathrm{~d} x_{k}+\sum_{k=1}^{\infty} \frac{\partial f}{\partial y_{k}} \mathrm{~d} y_{k} .
$$

As a result of a continuity condition, the coefficients of the differential twoform in the generating relation (10) are meromorphic.

First we will give a direct derivation of the Lax-Sato equations of generalized two-component d2DTL hierarchy from the generating relation (10). It is also possible to give a derivation based on an intermediate general statement about linear operators of the hierarchy, similar to the works [6], [4], but here we prefer to demonstrate a more straightforward way of exploiting the generating relation (10).

Taking a term of the generating relation containing $d \lambda \wedge d x_{n}$, we get

$$
\begin{aligned}
& \left(\left(J_{0}\right)^{-1}\left(\partial_{\lambda} \Lambda \partial_{n}^{+} M-\partial_{\lambda} M \partial_{n}^{+} \Lambda\right) \mathrm{d} \lambda \wedge \mathrm{d} x_{n}\right)^{\text {out }} \\
& \quad=\left(\left(J_{0}\right)^{-1}\left(\partial_{\lambda} \Lambda \partial_{n}^{+} M-\partial_{\lambda} M \partial_{n}^{+} \Lambda\right) \mathrm{d} \lambda \wedge \mathrm{d} x_{n}\right)^{\text {in }}
\end{aligned}
$$

where we introduce a notation $\partial_{n}^{+}=\frac{\partial}{\partial x_{n}}, \partial_{n}^{-}=\frac{\partial}{\partial y_{n}}$. Thus, taking into account (8), (9), we come to the conclusion that the functions

$$
A_{n}^{+}=\lambda\left(J_{0}\right)^{-1}\left(\partial_{\lambda} \Lambda \partial_{n}^{+} M-\partial_{\lambda} M \partial_{n}^{+} \Lambda\right)
$$

are polynomials, and they can be expressed by the formula

$$
A_{n}^{+}=\left(\left(J_{0}\right)^{-1}\left(\lambda \partial_{\lambda} \Lambda\right) \mathrm{e}^{(n+1) \Lambda}\right)_{+}^{\text {out }},
$$

where the subscripts ${ }_{+}$, - denote projection operators, $\left(\sum_{-\infty}^{\infty} u_{n} p^{n}\right)_{+}=$ $\sum_{n=0}^{\infty} u_{n} p^{n},\left(\sum_{-\infty}^{\infty} u_{n} p^{n}\right)_{-}=\sum_{-\infty}^{n=-1} u_{n} p^{n}$. In a similar way, taking a term of the generating relation containing $\mathrm{d} t \wedge \mathrm{d} x_{n}$, we conclude that the functions

$$
B_{n}^{+}=\left(J_{0}\right)^{-1}\left(\partial_{t} \Lambda \partial_{n}^{+} M-\partial_{t} M \partial_{n}^{+} \Lambda\right)
$$


are also polynomials, and they can be expressed by the formula

$$
B_{n}^{+}=\left(\left(J_{0}\right)^{-1}\left(\partial_{t} \Lambda\right) \mathrm{e}^{(n+1) \Lambda}\right)_{+}^{\text {out }},
$$

Resolving (12), (14) as linear equations with respect to $\partial_{n}^{+} \Lambda, \partial_{n}^{+} M$, we obtain Lax-Sato equations for the times $x_{n}$,

$$
\partial_{n}^{+}\left(\begin{array}{c}
\Lambda \\
M
\end{array}\right)=\left(A_{n}^{+} \partial_{t}-B_{n}^{+} \lambda \partial_{\lambda}\right)\left(\begin{array}{c}
\Lambda \\
M
\end{array}\right)
$$

Taking the terms of the generating relation containing $\mathrm{d} \lambda \wedge \mathrm{d} y_{n}, \mathrm{~d} t \wedge \mathrm{d} y_{n}$, we obtain Lax-Sato equations for the times $y_{n}$,

$$
\partial_{n}^{-}\left(\begin{array}{c}
\Lambda \\
M
\end{array}\right)=\left(A_{n}^{-} \partial_{t}-B_{n}^{-} \lambda \partial_{\lambda}\right)\left(\begin{array}{c}
\Lambda \\
M
\end{array}\right)
$$

where

$$
\begin{aligned}
& A_{n}^{-}=\left(\left(J_{0}\right)^{-1}\left(\lambda \partial_{\lambda} \Lambda\right) \mathrm{e}^{-(n+1) \Lambda}\right)_{-}^{\text {in }}, \\
& B_{n}^{-}=\left(\left(J_{0}\right)^{-1}\left(\partial_{t} \Lambda\right) \mathrm{e}^{-(n+1) \Lambda}\right)_{-}^{\text {in }} .
\end{aligned}
$$

The compatibility of the flows defined by the Lax-Sato equations can be proved similar to the case of Dunajski hierarchy [6], see also [5]. In explicit form, a complete set of Lax-Sato equations reads

$$
\begin{aligned}
& \left(\frac{\partial_{n}^{+}}{n+1}-\left(\frac{\lambda\left(\mathrm{e}^{(n+1) \Lambda}\right)_{\lambda}}{\{\Lambda, M\}}\right)_{+}^{\text {out }} \partial_{t}+\left(\frac{\left(\mathrm{e}^{(n+1) \Lambda}\right)_{t}}{\{\Lambda, M\}}\right)_{+}^{\text {out }} \lambda \partial_{\lambda}\right)\left(\begin{array}{c}
\Lambda \\
M
\end{array}\right)=0 \\
& \left(\frac{\partial_{n}^{-}}{n+1}+\left(\frac{\lambda\left(\mathrm{e}^{-(n+1) \Lambda}\right)_{\lambda}}{\{\Lambda, M\}}\right)_{-}^{\text {in }} \partial_{t}-\left(\frac{\left(\mathrm{e}^{-(n+1) \Lambda^{-}}\right)_{t}}{\{\Lambda, M\}}\right)_{-}^{\text {in }} \lambda \partial_{\lambda}\right)\left(\begin{array}{c}
\Lambda \\
M
\end{array}\right)=0
\end{aligned}
$$

where the definition of the Poisson bracket is $\{f, g\}=\lambda\left(f_{\lambda} g_{t}-f_{t} g_{\lambda}\right)$. LaxSato equations for the times $x=x_{1}, y=y_{1}, \partial_{1}^{+}=\partial_{x}, \partial_{1}^{-}=\partial_{y}$,

$$
\begin{aligned}
& \partial_{x} \boldsymbol{\Psi}=\left(\left(\lambda+\left(m_{1}^{+}\right)_{t}-l_{1}^{+}\right) \partial_{t}-\lambda l_{1}^{+} \partial_{\lambda}\right) \boldsymbol{\Psi}, \\
& \partial_{y} \boldsymbol{\Psi}=\left(\frac{1}{\lambda} \frac{\mathrm{e}^{-\phi}}{m_{t}} \partial_{t}+\frac{\left(\mathrm{e}^{-\phi}\right)_{t}}{m_{t}} \partial_{\lambda}\right) \boldsymbol{\Psi},
\end{aligned}
$$

where $\Psi=\left(\begin{array}{c}\Lambda \\ M\end{array}\right), m=m_{0}+t$, correspond to the Lax pair (6), where the coefficients in the first Lax-Sato equation can be transformed to the form (6) 
by taking its expansion at $\lambda=0$, and the system (5) arises as a compatibility condition.

Lax-Sato equations $(16,17)$ define the evolution of the series $\Lambda^{\text {in }}, \Lambda^{\text {out }}$, $M^{\text {in }}, M^{\text {out }}$. The only term containing an interaction between $\Lambda$ and $M$ is $\{\Lambda, M\}$. The condition $\{\Lambda, M\}=1$ splits out equations for $\Lambda$ and reduces the hierarchy $(16,17)$ to the d2DTL hierarchy, while the condition $\Lambda=\ln \lambda$ - to the hierarchy, considered by Martínez Alonso and Shabat [10, 11], see also Pavlov [3].

\subsection{The dressing scheme}

A dressing scheme for the generalized two-component d2DTL hierarchy can be formulated in terms of the two-component nonlinear Riemann-Hilbert problem on the unit circle $S$ in the complex plane of the variable $\lambda$,

$$
\begin{aligned}
& \Lambda^{\text {out }}=F_{1}\left(\Lambda^{\text {in }}, M^{\text {in }}\right), \\
& M^{\text {out }}=F_{2}\left(\Lambda^{\text {in }}, M^{\text {in }}\right),
\end{aligned}
$$

where the functions $\Lambda^{\text {out }}(\lambda, \mathbf{x}, \mathbf{y}, t), M^{\text {out }}(\lambda, \mathbf{x}, \mathbf{y}, t)$ are defined inside the unit circle, the functions $\Lambda^{\text {in }}(\lambda, \mathbf{x}, \mathbf{y}, t), M^{\text {in }}(\lambda, \mathbf{x}, \mathbf{y}, t)$ outside the unit circle by the series of the form (8), (9), with $\Lambda^{\text {in }}-\ln \lambda, M^{\text {in }}-M_{0}^{\text {in }}$ analytic in the unit disc, and $\Lambda^{\text {out }}-\ln \lambda, M^{\text {out }}-M_{0}^{\text {out }}$ analytic outside the unit disc and decreasing at infinity. The functions $F_{1}, F_{2}$ are suggested to define (at least locally) a diffeomorphism of the plane, $\mathbf{F} \in \operatorname{Diff}(2)$, and we call them the dressing data. Let us consider a differential form

$$
\Omega=\mathrm{d} \Lambda \wedge \mathrm{d} M
$$

The condition for this form on the unit circle is determined by the Jacobian of the diffeomorphism defined by $F_{1}, F_{2}$,

$$
\Omega^{\text {out }}=\left|\frac{D\left(F_{1}, F_{2}\right)}{D\left(\Lambda^{\text {in }}, M^{\text {in }}\right)}\right| \Omega^{-},
$$

where for the Jacobian we use a notation

$$
\left|\frac{D(f, g)}{D(x, y)}\right|=\operatorname{det} \frac{D(f, g)}{D(x, y)}=\operatorname{det}\left(\begin{array}{ll}
\frac{\partial f}{\partial x} & \frac{\partial f}{\partial y} \\
\frac{\partial g}{\partial x} & \frac{\partial g}{\partial y}
\end{array}\right) .
$$

Expressing the differential $\mathrm{d}$ in terms of independent variables $\lambda, \mathbf{x}, \mathbf{y}, t(11)$, we come to the conclusion that all the coefficients of the differential twoform $\Omega$ in terms of these variables transform according to the condition (19). 
Normalizing the form by one of the coefficients, we obtain the differential form continuous on the unit circle. Taking the coefficient corresponding to $d \lambda \wedge \mathrm{d} t$, we obtain the relation

$$
\left(\left|\frac{D(\Lambda, M)}{D(\lambda, t)}\right|^{-1} \Omega\right)^{\text {out }}=\left(\left|\frac{D(\Lambda, M)}{D(\lambda, t)}\right|^{-1} \Omega\right)^{\text {in }},
$$

which is equivalent to the generating relation (10) after multiplication by $\lambda^{-1}$

$$
J_{0}=\lambda\left|\frac{D(\Lambda, M)}{D(\lambda, t)}\right|=\operatorname{det}\left(\begin{array}{cc}
\lambda \partial_{\lambda} \Lambda & \partial_{t} \Lambda \\
\lambda \partial_{\lambda} M & \partial_{t} M
\end{array}\right)
$$

\subsection{Differential reductions}

Recently we have introduced a class of reductions of the Manakov-Santini hierarchy [5] connected with the interpolating system [12]. Similar reductions can be constructed for the generalized two-component d2DTL hierarchy, we are going to study them in detail elsewhere. Here we will only present the simplest reduction, which is analogous to the reduction of the Manakov-Santini system leading to the interpolating system [12]. The reduced hierarchy is defined by the relation

$$
(\exp (-\alpha \Lambda) \mathrm{d} \Lambda \wedge \mathrm{d} M)^{\text {out }}=(\exp (-\alpha \Lambda) \mathrm{d} \Lambda \wedge \mathrm{d} M)^{\mathrm{in}}
$$

where $\alpha$ is a parameter ( $\alpha=0$ corresponds to the case of d2DTL hierarchy), which implies that

$$
J_{0}=\lambda^{-\alpha} \exp (\alpha \Lambda)
$$

and in terms of the system (5) we get a reduction

$$
\mathrm{e}^{\alpha \phi}=m_{t} .
$$

This reduction makes it possible to rewrite the system (5) as one equation for $m$,

$$
m_{t t}=\left(m_{t}\right)^{\frac{1}{\alpha}}\left(m_{t y} m_{x}-m_{x y} m_{t}\right),
$$

or in the form of deformed d2DTL equation,

$$
\begin{aligned}
& \left(\mathrm{e}^{-\phi}\right)_{t t}=m_{t} \phi_{x y}-m_{x} \phi_{t y}, \\
& m_{t}=\mathrm{e}^{\alpha \phi} .
\end{aligned}
$$




\section{Transformations and a symmetric generalization}

The system (5) we have introduced above doesn't preserve the symmetry of the dispersionless 2DTL equation (7) with respect to the variables $x, y$. To introduce a symmetric generalization of the equation (7) and its elliptic version

$$
\left(\mathrm{e}^{-\phi}\right)_{t t}=\phi_{z \bar{z}}
$$

it is possible to change the form of the series for $\Lambda, M$ to have an explicit symmetry between zero and infinity in the complex plane of the spectral variable $\lambda$, then the generating relation (10) will lead to symmetric LaxSato equations. However, we prefer to consider first the transformations of the hierachy that will allow us to transfer to the symmetric case and will give the connection between different generalizations of the d2DTL equation.

First, there is a gauge transformation, present already in d2DTL case $[7,8]$, which changes the Lax pair, but preserves the equations

$$
\lambda \rightarrow \lambda \exp (-\epsilon \phi)
$$

where $\epsilon$ is a parameter. After this transformation we get $\Lambda$ of the form

$$
\begin{aligned}
& \Lambda^{\text {out }}=\ln \lambda-\epsilon \phi+\sum_{k=1}^{\infty} l_{k}^{+} \lambda^{-k}, \\
& \Lambda^{\text {in }}=\ln \lambda+(1-\epsilon) \phi+\sum_{k=1}^{\infty} l_{k}^{-} \lambda^{k} .
\end{aligned}
$$

In the Lax pair one should perform a substitution

$$
\begin{aligned}
& \lambda \rightarrow \lambda \exp (-\epsilon \phi), \partial_{\lambda} \rightarrow \exp (\epsilon \phi) \partial_{\lambda} . \\
& \partial_{x} \rightarrow \partial_{x}+\epsilon \lambda \phi_{x} \partial_{\lambda}, \partial_{y} \rightarrow \partial_{y}+\epsilon \lambda \phi_{y} \partial_{\lambda}, \partial_{t} \rightarrow \partial_{t}+\epsilon \lambda \phi_{t} \partial_{\lambda},
\end{aligned}
$$

In the elliptic d2DTL case (21) for $\epsilon=\frac{1}{2}$ we get a symmetric Lax pair

$$
\begin{aligned}
& \partial_{z} \boldsymbol{\Psi}=L_{1} \boldsymbol{\Psi}=\left(\left(\lambda \mathrm{e}^{-\frac{1}{2} \phi}\right) \partial_{t}+\frac{1}{2}\left(\phi_{z}+\lambda \mathrm{e}^{-\frac{1}{2} \phi} \phi_{t}\right) \lambda \partial_{\lambda}\right) \boldsymbol{\Psi} \\
& \partial_{\bar{z}} \boldsymbol{\Psi}=L_{2} \boldsymbol{\Psi}=\left(\left(\frac{1}{\lambda} \mathrm{e}^{-\frac{1}{2} \phi}\right) \partial_{t}-\frac{1}{2}\left(\phi_{\bar{z}}+\lambda \mathrm{e}^{-\frac{1}{2} \phi} \phi_{t}\right) \lambda \partial_{\lambda}\right) \boldsymbol{\Psi}
\end{aligned}
$$

and on the unit circle $L_{1}=\bar{L}_{2}$. 
To get a symmetric two-component generalization of the ellyptic d2DTL equation and a symmetric Lax pair for it, we should also use a hodograph type transformation

$$
t=\tau-\alpha m_{0}
$$

(where $\tau$ is a new 'time', $\alpha$ is a parameter), which gives $M$ of the form

$$
\begin{aligned}
& M^{\text {out }}=M_{0}^{\text {out }}+(1-\alpha) m_{0}+\sum_{k=1}^{\infty} m_{k}^{+} \mathrm{e}^{-k \Lambda^{+}}, \\
& M^{\text {in }}=M_{0}^{\text {in }}-\alpha m_{0}+\sum_{k=1}^{\infty} m_{k}^{-} \mathrm{e}^{k \Lambda^{+}} \\
& M_{0}=\tau+x \mathrm{e}^{\Lambda}+y \mathrm{e}^{-\Lambda}+\ldots
\end{aligned}
$$

Derivatives transform as follows,

$\partial_{x} \rightarrow \partial_{x}+\frac{\alpha m_{0 x}}{1-\alpha m_{0 \tau}} \partial_{\tau}, \partial_{y} \rightarrow \partial_{y}+\frac{\alpha m_{0 y}}{1-\alpha m_{0 \tau}} \partial_{\tau}, \partial_{t} \rightarrow \partial_{\tau}+\frac{\alpha m_{0 \tau}}{1-\alpha m_{0 \tau}} \partial_{\tau}$.

Applying these transformations to the system (5), where $m=m_{0}+t$, we obtain a one-parametric family of two-component generalizations of the d2DTL equation.

Taking $x=z, y=\bar{z}, \epsilon=\frac{1}{2}, \phi \rightarrow-2 \varphi, \alpha=\frac{1}{2}, m_{0}=-2 \mathrm{i} \mu$, we get

$$
\begin{aligned}
& \Lambda^{\text {out }}=\ln \lambda+\varphi+\sum_{k=1}^{\infty} l_{k}^{+} \lambda^{-k}, \quad \Lambda^{\text {in }}=\ln \lambda-\varphi+\sum_{k=1}^{\infty} l_{k}^{-} \lambda^{k} \\
& M^{\text {out }}=M_{0}^{\text {out }}+\mathrm{i} \mu+\sum_{k=1}^{\infty} m_{k}^{+} \mathrm{e}^{-k \Lambda}, \quad M^{\text {in }}=M_{0}^{\text {in }}-\mathrm{i} \mu+\sum_{k=1}^{\infty} m_{k}^{-} \mathrm{e}^{k \lambda}, \\
& M_{0}=\tau+z \mathrm{e}^{\Lambda}+\bar{z} \mathrm{e}^{-\Lambda}+\ldots
\end{aligned}
$$

where for the case of ellyptic d2DTL we suggest that $\mu, \varphi$ are real, and on the unit circle $\lambda \bar{\lambda}=1$

$$
\begin{aligned}
& M^{\text {out }}=\bar{M}^{\text {in }}, \\
& \Lambda^{\text {out }}=-\bar{\Lambda}^{\text {in }} .
\end{aligned}
$$

From the Lax pair (6) we obtain a symmetric Lax pair

$$
\begin{array}{ll}
\partial_{z} \boldsymbol{\Psi}=L_{1} \boldsymbol{\Psi}, & L_{1}=\left(\lambda \mathrm{e}^{\varphi} u+v\right) \partial_{\tau}+\left(\left(\varphi_{\tau} v-\varphi_{z}\right)-\lambda u \mathrm{e}^{\varphi} \varphi_{\tau}\right) \lambda \partial_{\lambda}, \\
\partial_{\bar{z}} \boldsymbol{\Psi}=L_{2} \boldsymbol{\Psi}, & L_{2}=\left(\frac{1}{\lambda} \mathrm{e}^{\varphi} \bar{u}+\bar{v}\right) \partial_{\tau}-\left(\left(\varphi_{\tau} \bar{v}-\varphi_{\bar{z}}\right)-\frac{1}{\lambda} \bar{u} \mathrm{e}^{\varphi} \varphi_{\tau}\right) \lambda \partial_{\lambda},
\end{array}
$$


on the unit circle $L_{1}=\bar{L}_{2}$,

$$
u=\frac{1}{1+\mathrm{i} \mu_{\tau}}, \quad v=\frac{-\mathrm{i} \mu_{z}}{1-\mathrm{i} \mu_{\tau}} .
$$

Equation (5) transforms to the symmetric two-component generalization of the ellyptic d2DTL equation (21),

$$
\begin{aligned}
& \left(v_{\bar{z}}+\mathrm{e}^{\varphi} u \partial_{\tau}\left(\mathrm{e}^{\varphi} \bar{u}\right)+v \partial_{\tau} \bar{v}\right)-\text { c.c. }=0, \\
& \left(\partial_{\bar{z}}\left(\varphi_{\tau} v-\varphi_{z}\right)+\mathrm{e}^{\varphi} u \partial_{\tau}\left(\bar{u} \mathrm{e}^{\varphi} \varphi_{\tau}\right)-v \partial_{\tau}\left(\varphi_{\tau} \bar{v}-\varphi_{\bar{z}}\right)+u \bar{u} \mathrm{e}^{2 \varphi} \varphi_{\tau} \varphi_{\tau}\right) \\
& \quad+\text { c.c. }=0
\end{aligned}
$$

If $\mu=0(u=1, v=0)$, the first equation vanishes, the second gives the d2DTL equation for $\phi=(-2 \varphi)$.

If $\varphi=0$, the second equation vanishes, the first gives

$$
\left(v_{\bar{z}}+u \partial_{\tau}(\bar{u})+v \partial_{\tau} \bar{v}\right)-\text { c.c. }=0,
$$

or, in explicit form,

$\mu_{\tau \tau}=\frac{1}{2}\left(\mu_{z} \mu_{\bar{z}}-\left(1+\mu_{\tau}^{2}\right)\right)^{-1}\left(\mu_{\tau}^{2}\left(\partial_{\tau}\left(\mu_{z} \mu_{\bar{z}}\right)-\mathrm{i}\left(\mu_{z \tau} \mu_{\bar{z}}-\mu_{z} \mu_{\bar{z} \tau}\right)\right)-\mu_{z \bar{z}}\left(1+\mu_{\tau}^{2}\right)\right)$.

\section{Acknowledgments}

The author is grateful to S.V. Manakov and P.M. Santini for useful discussions. This research was partially supported by the Russian Foundation for Basic Research under grants no. 10-01-00787, 09-01-92439, and by the President of Russia grant 4887.2008.2 (scientific schools).

\section{References}

[1] S. V. Manakov and P. M. Santini, The Cauchy problem on the plane for the dispersionless Kadomtsev-Petviashvili equation, JETP Lett. 83 (2006) 462-6.

[2] S. V. Manakov and P. M. Santini, A hierarchy of integrable PDEs in $2+1$ dimensions associated with 2-dimensional vector fields, Theor. Math. Phys. 152 (2007) 1004-1011.

[3] M.V. Pavlov, Integrable hydrodynamic chains, J. Math. Phys. 44(9) (2003) 4134-4156 
[4] L.V. Bogdanov, A class of multidimensional integrable hierarchies and their reductions, Theoretical and Mathematical Physics 160(1) (2009) $887-893$

[5] L. V. Bogdanov, On a class of reductions of the Manakov-Santini hierarchy connected with the interpolating system, J Phys. A: Math. Theor., to be published

[6] L. V. Bogdanov, V. S. Dryuma and S. V. Manakov, Dunajski generalization of the second heavenly equation: dressing method and the hierarchy, J Phys. A: Math. Theor. 40 (2007) 14383-14393.

[7] K. Takasaki and T. Takebe, SDiff(2) Toda equation - Hierarchy, Tau function, and symmetries, Letters in Mathematical Physics 23(3), 205214 (1991)

[8] K. Takasaki and T. Takebe, Integrable Hierarchies and Dispersionless Limit, Reviews in Mathematical Physics 7(05), 743-808 (1995)

[9] S. V. Manakov and P. M. Santini, The dispersionless 2D Toda equation: dressing, Cauchy problem, longtime behaviour, implicit solutions and wave breaking, Journal of Physics A: Mathematical and Theoretical 42(9), 095203 (2009)

[10] L. Martínez Alonso and A. B. Shabat, Energy-dependent potentials revisited: a universal hierarchy of hydrodynamic type, Phys. Lett. A 300 (2002) 58-64

[11] L. Martínez Alonso and A. B. Shabat, Hydrodynamic reductions and solutions of a universal hierarchy, Theor. Math. Phys. 140 (2004) 10731085

[12] Maciej Dunajski, An interpolating dispersionless integrable system, J. Phys. A: Math. Theor. 41 (2008) 315202 